\title{
Spatial diquark correlations in a hadron
}

\author{
Jeremy Green* and John Negele \\ Center for Theoretical Physics, Massachusetts Institute of Technology, Cambridge, MA 02139 \\ E-mail: jrgreen@mit.edu
}

\section{Michael Engelhardt}

Department of Physics, New Mexico State University, Las Cruces, NM 88003-0001

\section{Patrick Varilly}

Department of Physics, University of California, Berkeley, Berkeley, CA 94720

\begin{abstract}
Using lattice QCD, a diquark can be studied in a gauge-invariant manner by binding it to a static quark in a heavy-light-light hadron. We compute the simultaneous two-quark density of a diquark, including corrections for periodic boundary conditions. We define a correlation function to isolate the intrinsic correlations of the diquark and reduce the effects caused by the presence of the static quark. Away from the immediate vicinity of the static quark, the diquark has a consistent shape, with much stronger correlations seen in the good (scalar) diquark than in the bad (vector) diquark. We present results for $m_{\pi}=293 \mathrm{MeV}$ in $N_{f}=2+1 \mathrm{QCD}$ as well as $m_{\pi}=940 \mathrm{MeV}$ in quenched $\mathrm{QCD}$, and discuss the dependence of the spatial size on the pion mass.
\end{abstract}

The XXVIII International Symposium on Lattice Field Theory

June 14-19,2010

Villasimius, Sardinia Italy

${ }^{*}$ Speaker. 


\section{Introduction}

Diquarks are two-quark systems. Collective behavior of a diquark has been invoked to explain many phenomena of strong interactions [1]. By introducing diquarks as effective degrees of freedom in chiral perturbation theory, they have been used to explain the enhancement of $\Delta I=\frac{1}{2}$ nonleptonic weak decays [2]. A simple quark-diquark model is quite successful at organizing the spectrum of excited light baryon states [3].

The simplest diquark operators are quark bilinears with spinor part $q^{T} C \Gamma q$. The favored combinations are color antitriplet, even parity [4]. These are divided into "good" and "bad" diquarks. The good diquarks, $q^{T} C \gamma_{5} q$, have spin 0 and are flavor antisymmetric due to fermion statistics. The bad diquarks, $q^{T} C \gamma_{i} q$, have spin 1 and are flavor symmetric.

Both one-gluon exchange in a quark model [5, 6] and instanton [7] models give a spin coupling energy proportional to $\vec{S}_{i} \cdot \vec{S}_{j}$, which favors the good diquark over the bad diquark. The strength of this coupling falls off with increasing quark masses. For the instanton model, the effective interaction has a flavor dependence that also favors the good diquark.

\section{Earlier studies in baryons}

Since diquarks are not color singlets, studying them within the framework of lattice QCD typically requires that they be combined with a third quark to form a color singlet. Diquark attractions result in spatial correlations between the two quarks in the diquark, which can be probed by computing a wavefunction or two-quark density.

In one study [8], using gauge fixing and three quarks of equal mass, a wavefunction was computed by displacing quarks at the sink,

$$
\begin{aligned}
\psi\left(\mathbf{r}_{1}, \mathbf{r}_{2}\right) \propto \sum_{\mathbf{r}_{s}}\left\langle u\left(\mathbf{r}_{s}+\mathbf{r}_{1}, t\right) d\left(\mathbf{r}_{s}+\mathbf{r}_{2}, t\right) s\left(\mathbf{r}_{s}, t\right)\right. \\
\\
\times \bar{u}(\mathbf{0}, 0) \bar{d}(\mathbf{0}, 0) \bar{s}(\mathbf{0}, 0)\rangle
\end{aligned}
$$

Then, using the more convenient coordinates

$$
\mathbf{R}=\left(\mathbf{r}_{1}+\mathbf{r}_{2}\right) / 2 \quad \text { and } \quad \mathbf{r}=\left(\mathbf{r}_{1}-\mathbf{r}_{2}\right) / 2
$$
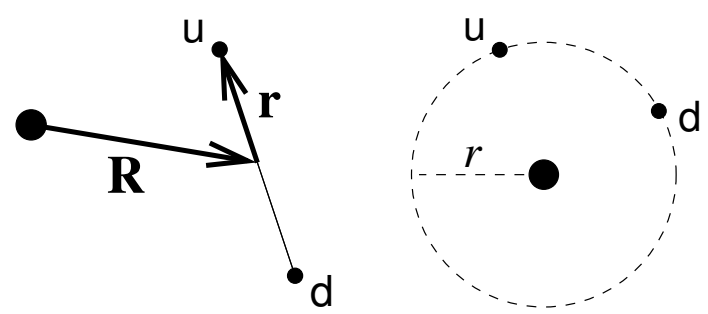

Figure 1: Geometry of the three quarks used in this paper and in [8] (left), and restricted geometry used in [9] (right).

(Fig. 1, left), the wavefunction of the good and bad diquarks was shown for different fixed $R=|\mathbf{R}|$ as a function of $\mathbf{r}$, in both Coulomb gauge and Landau gauge. In all cases, the wave function had a peak near $\mathbf{r}=0$, but it was found to fall off more rapidly for the good diquark, consistent with the expectation that good diquarks are more tightly bound.

In a second study [9], spatial correlations were investigated by computing the two quark density $\rho_{2}\left(\mathbf{r}_{u}, \mathbf{r}_{d}\right)$ for a $(u, d)$ diquark in the background of a static quark. To isolate correlations caused by the diquark interaction, analysis was restricted to spherical shells $\left|\mathbf{r}_{u}\right|=\left|\mathbf{r}_{d}\right|=r$ (Fig. 1, right). For both good and bad diquarks, the density was found to be concentrated near $r_{u d}=\left|\mathbf{r}_{u}-\mathbf{r}_{d}\right|=0$, and the effect was much stronger for the good diquark. Fitting $\rho_{2}$ for the good diquark to $\exp \left(-r_{u d} / r_{0}(r)\right), r_{0}$ reached a plateau for large $r$, giving a characteristic size $r_{0}=1.1 \pm 0.2 \mathrm{fm}$. 


\section{Correlation function}

In the first study, the wavefunctions of good and bad diquarks were compared for an unrestricted geometry, but they were not compared against an uncorrelated wavefunction. In the second study, the intrinsic clustering caused by the diquark interaction was shown, however this was achieved by using a restricted geometry.

To overcome these limitations, we combined a diquark with a static quark, using the baryon operator $B=\varepsilon^{a b c}\left(u_{a}^{T} C \Gamma d_{b}\right) s_{c}$, taking $\Gamma=\gamma_{5}$ for the good diquark and $\Gamma=\gamma_{1}$ for the bad diquark, and calculated the single quark density and the simultaneous two-quark density:

$$
\rho_{1}(\mathbf{r})=N_{1} \frac{\left\langle 0\left|B\left(\mathbf{0}, t_{f}\right) J_{0}^{u}(\mathbf{r}, t) \bar{B}\left(\mathbf{0}, t_{i}\right)\right| 0\right\rangle}{\left\langle 0\left|B\left(\mathbf{0}, t_{f}\right) \bar{B}\left(\mathbf{0}, t_{i}\right)\right| 0\right\rangle}, \quad \rho_{2}\left(\mathbf{r}_{1}, \mathbf{r}_{2}\right)=N_{2} \frac{\left\langle 0\left|B\left(\mathbf{0}, t_{f}\right) J_{0}^{u}\left(\mathbf{r}_{1}, t\right) J_{0}^{d}\left(\mathbf{r}_{2}, t\right) \bar{B}\left(\mathbf{0}, t_{i}\right)\right| 0\right\rangle}{\left\langle 0\left|B\left(\mathbf{0}, t_{f}\right) \bar{B}\left(\mathbf{0}, t_{i}\right)\right| 0\right\rangle} .
$$

Here, there are insertions of the current $J_{\mu}^{f}=\bar{f} \gamma_{\mu} f$, and the normalization factors $N_{1,2}$ are required since this local current is not conserved on the lattice.

In a system where $\rho_{1}(\mathbf{r})$ is uniform, the two-particle correlation can be defined as

$$
C_{0}\left(\mathbf{r}_{1}, \mathbf{r}_{2}\right)=\rho_{2}\left(\mathbf{r}_{1}, \mathbf{r}_{2}\right)-\rho_{1}\left(\mathbf{r}_{1}\right) \rho_{1}\left(\mathbf{r}_{2}\right) .
$$

Deviations from zero are seen as evidence for interactions between particles. This correlation integrates to zero and approaches zero as the relative distance $r_{12}=\left|\mathbf{r}_{1}-\mathbf{r}_{2}\right|$ increases beyond the range of interactions in the system.

The situation considered here is not so simple. The single particle density is not uniform: it is concentrated near the static quark. $C_{0}$ will still integrate to zero and fall off at large distances, however it is also larger near the static quark and this obscures the diquark correlations.

In order to remove the effect of the static quark, we define the normalized correlation function:

$$
C\left(\mathbf{r}_{1}, \mathbf{r}_{2}\right)=\frac{\rho_{2}\left(\mathbf{r}_{1}, \mathbf{r}_{2}\right)-\rho_{1}\left(\mathbf{r}_{1}\right) \rho_{1}\left(\mathbf{r}_{2}\right)}{\rho_{1}\left(\mathbf{r}_{1}\right) \rho_{1}\left(\mathbf{r}_{2}\right)}
$$

This divides out the tendency to stay near the static quark and retains the property of being zero if the two light quarks are uncorrelated (i.e. if $\left.\rho_{2}\left(\mathbf{r}_{1}, \mathbf{r}_{2}\right)=\rho_{1}\left(\mathbf{r}_{1}\right) \rho_{1}\left(\mathbf{r}_{2}\right)\right)$. The downsides are that $C$ no longer integrates to zero, and it is possible for $C(\mathbf{r}, \mathbf{r})$ to increase without bound as $|\mathbf{r}| \rightarrow \infty$.

\section{Density in a periodic box}

We assume the lattice spacing is small enough that in an infinite volume we can treat $\rho_{2}\left(\mathbf{r}_{1}, \mathbf{r}_{2}\right)$ as a function of $R=|\mathbf{R}|, r=|\mathbf{r}|$, and $\theta$, the angle between $\mathbf{R}$ and $\mathbf{r}$. Since the calculation is actually carried out on a finite lattice volume, to recover the infinite volume result, we need to deal with the effect of periodic boundary conditions.

The problem of dealing with $\rho$ in periodic boundary conditions has been previously analyzed for the case of a meson [10]. It was found that $\rho_{1}(\mathbf{r})=\sum_{\mathbf{n} \in \mathbb{Z}^{3}} \tilde{\rho}_{1}(\mathbf{r}+\mathbf{n} L)$, where $\tilde{\rho}_{1}$ differs from the infinite volume result only for $r \gtrsim L$ due to interactions with periodic images.

For this study, we have a baryon, and there is an additional complication: the contribution from "exchange diagrams" in which the two quarks travel in opposite directions across the periodic 
boundary and can form a color singlet. As the lattice size grows, this becomes dominated by the propagation of the lightest meson and so falls off as $\exp \left(-m_{\pi} L\right)$.

Ignoring the exchange diagrams and interactions with periodic images we find

$$
\rho_{2}\left(\mathbf{r}_{1}, \mathbf{r}_{2}\right)=\sum_{\mathbf{n}_{1}, \mathbf{n}_{2} \in \mathbb{Z}^{3}} \rho_{2}^{\prime}\left(\mathbf{r}_{1}+\mathbf{n}_{1} L, \mathbf{r}_{2}+\mathbf{n}_{2} L\right)
$$

where $\rho_{2}^{\prime}$ is the infinite volume two quark density. In order to deal with image effects, a phenomenological fit is used. Given a good functional form $f_{2}^{\prime}\left(\mathbf{r}_{1}, \mathbf{r}_{2}\right)$ for $\rho_{2}^{\prime}$ (invariant under simultaneous rotations of $\mathbf{r}_{1}$ and $\mathbf{r}_{2}$ as well as exchange of $\mathbf{r}_{1}$ and $\mathbf{r}_{2}$ ), the nearest images are added in:

$$
f_{2}\left(\mathbf{r}_{1}, \mathbf{r}_{2}\right)=\sum_{n_{1}^{i}, n_{2}^{j} \in\{-1,0,1\}} f_{2}^{\prime}\left(\mathbf{r}_{1}+\mathbf{n}_{1} L, \mathbf{r}_{2}+\mathbf{n}_{2} L\right) .
$$

This function and its lattice integral $f_{1}(\mathbf{r})=\sum_{\mathbf{r}_{2}} f_{2}\left(\mathbf{r}, \mathbf{r}_{2}\right)$ can be simultaneously fit to $\rho_{2}$ and $\rho_{1}$ using a nonlinear weighted least squares method. This allows the images to be subtracted off, giving $\rho_{2}^{\prime} \simeq \rho_{2}-f_{2}+f_{2}^{\prime}$ and $\rho_{1}^{\prime} \simeq \rho_{1}-f_{1}+f_{1}^{\prime}$, where $f_{1}^{\prime}(\mathbf{r})=\int d^{3} \mathbf{r}_{2} f_{2}^{\prime}\left(\mathbf{r}, \mathbf{r}_{2}\right)$.

The so-called $\Delta$ ansatz for the static potential for interacting quarks [11, 12] was used as motivation for the functional form of $f_{2}^{\prime}$. We ultimately found that the following eleven parameter functional form gave a reasonably good fit:

$$
\begin{gathered}
f_{2}^{\prime}\left(\mathbf{r}_{1}, \mathbf{r}_{2}\right)=A g\left(r_{1}, B, a_{1}, 0\right) g\left(r_{2}, B, a_{1}, 0\right) g\left(r, C, a_{2}, b_{2}\right) e^{-D\left(r_{1}^{3 / 2}+r_{2}^{3 / 2}\right)+E r^{3 / 2}+F R^{3 / 2}+G e^{-\alpha \sqrt{r_{1}^{2}+r_{2}^{2}}},} \\
\text { with } g(r, A, a, b)=\left\{\begin{array}{ll}
\exp (-A r) & r>a \\
c_{1}-b r-c_{2} r^{2} & r<a
\end{array},\right.
\end{gathered}
$$

where $c_{1,2}$ are given by the requirement that $g$ and $\frac{\partial g}{\partial r}$ are continuous at $r=a$.

\section{Lattice Calculations}

We used a mixed action scheme [13] with domain wall valence quarks on an asqtad sea, with $m_{\pi}=293(1) \mathrm{MeV}$ and $a=0.1241(25) \mathrm{fm}$. This ensemble had $453 \mathrm{HYP}$ smeared MILC gauge configurations [14], which have $N_{f}=2+1$ and volume $20^{3} \times 64$. Propagators were computed every 8 lattice units in the time direction, allowing for 8 measurements per gauge configuration, with source and sink separated by 8 lattice units. Wilson lines were computed using HYP smeared gauge links, and measurements were averaged over seven positions for the static quark: $\mathbf{x}=\mathbf{0}$ and the six nearest neighbors.

For comparison, we also used a heavy quark mass, with $m_{\pi} \approx 940 \mathrm{MeV}$. Since the effects of dynamical sea quarks are negligible at that mass, we performed a calculation with $\kappa=0.153$ Wilson fermions on 200 configurations from the OSU_Q60a ensemble [15], which are $16^{3} \times 32$ with quenched $\beta=6.00$ Wilson action. From the static quark potential, this has $a / r_{0}=0.186$ [16]. Using $r_{0}=0.47 \mathrm{fm}$, the lattice spacing is $a=0.088 \mathrm{fm}$. We used a source-sink separation of 11 lattice units and averaged measurements over the two central timeslices.

The functions $f_{1,2}$ were fit to a restricted set of the lattice measurements $\rho_{1,2}$. Three conditions were imposed to reduce the influence of the points most affected by images: $r<8 a$ for $\rho_{1}, r_{1}^{2}+r_{2}^{2}<$ 

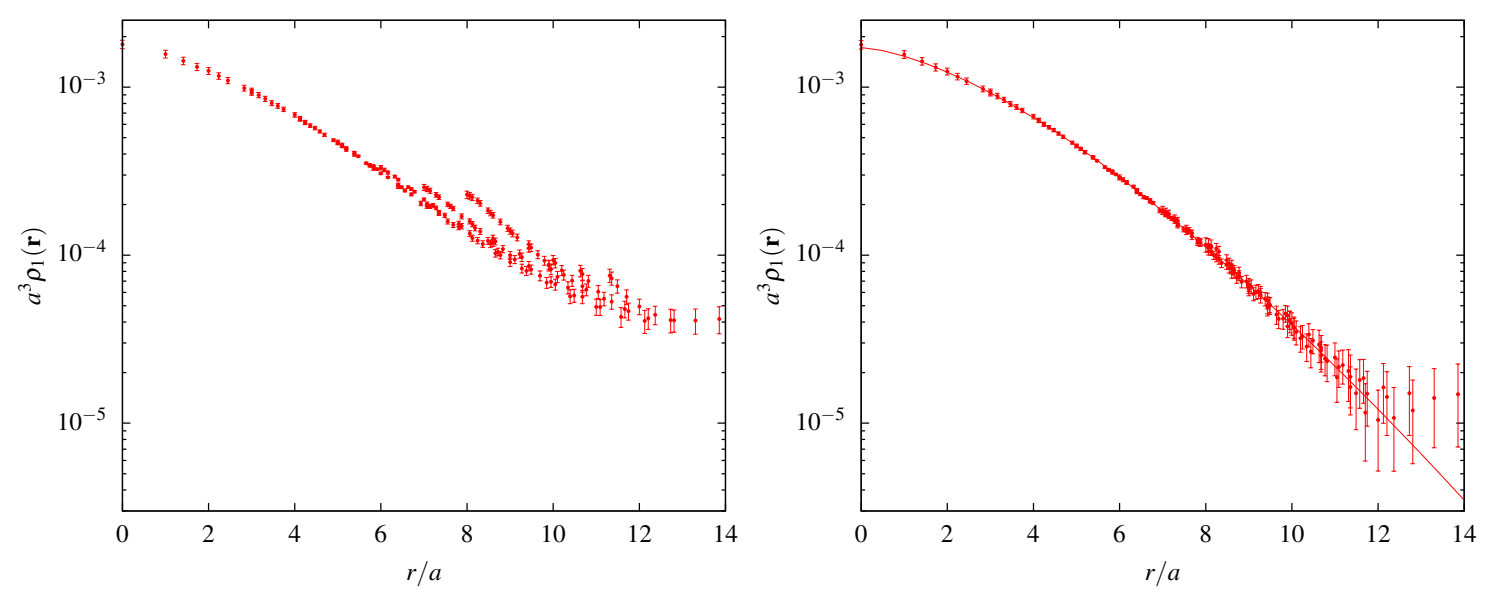

Figure 2: $\rho_{1}(\mathbf{r})$ without (left) and with (right) image corrections for the good diquark on the quenched $m_{\pi}=940 \mathrm{MeV}$ ensemble
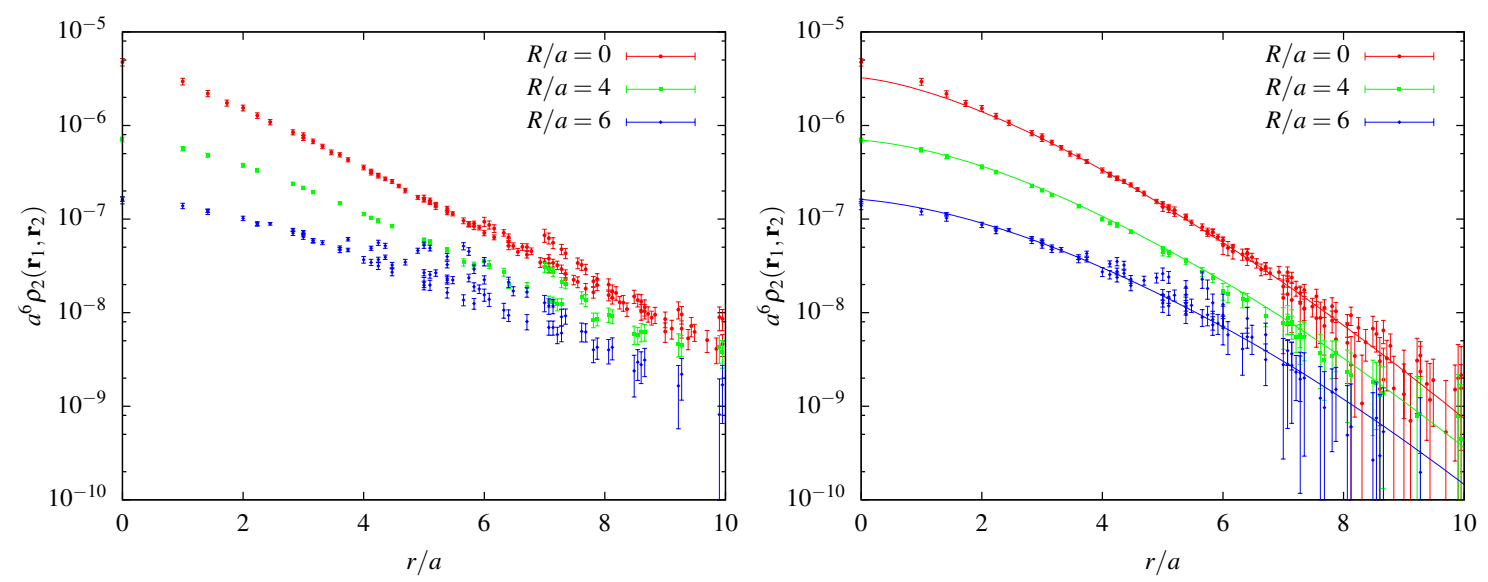

Figure 3: $\rho_{2}\left(\mathbf{r}_{1}, \mathbf{r}_{2}\right)$ without (left) and with (right) image corrections for the good diquark on the quenched $m_{\pi}=940 \mathrm{MeV}$ ensemble, as a function of $r$ with $\mathbf{r} \perp \mathbf{R}$ and $R / a=0,4,6$.

$100 a^{2}$ for $\rho_{2}$, and in both cases $r_{\text {image }} \geq 11 a$, where $r_{\text {image }}$ is the distance to the nearest periodic image of the static quark. Fits had $\chi^{2}$ per degree of freedom ranging from 0.25 to 1.85 .

In the quenched good diquark case, Figure 2 shows the effect of image corrections for $\rho_{1}$, and here this procedure is quite successful, even extrapolating beyond the range included in the fit.

For $\rho_{2}$, the fit isn't as good as for $\rho_{1}$, but it still works well. Figure 3 shows $\rho_{2}$ with and without image corrections. The figure on the right looks cleaner for two reasons. First, the fit function is determined using a global fit, which allows for small deviation from the specified $R$ and $\theta$ to be compensated for. Second, image corrections have been applied, which are substantial for points distant from the origin. The end result is that the difference between the plotted point and the fit curve is equal to the difference between the raw data point and the fit for that point.

\section{Results and discussion}

As a check of how well the correlation function isolates the diquark from the effect of the 

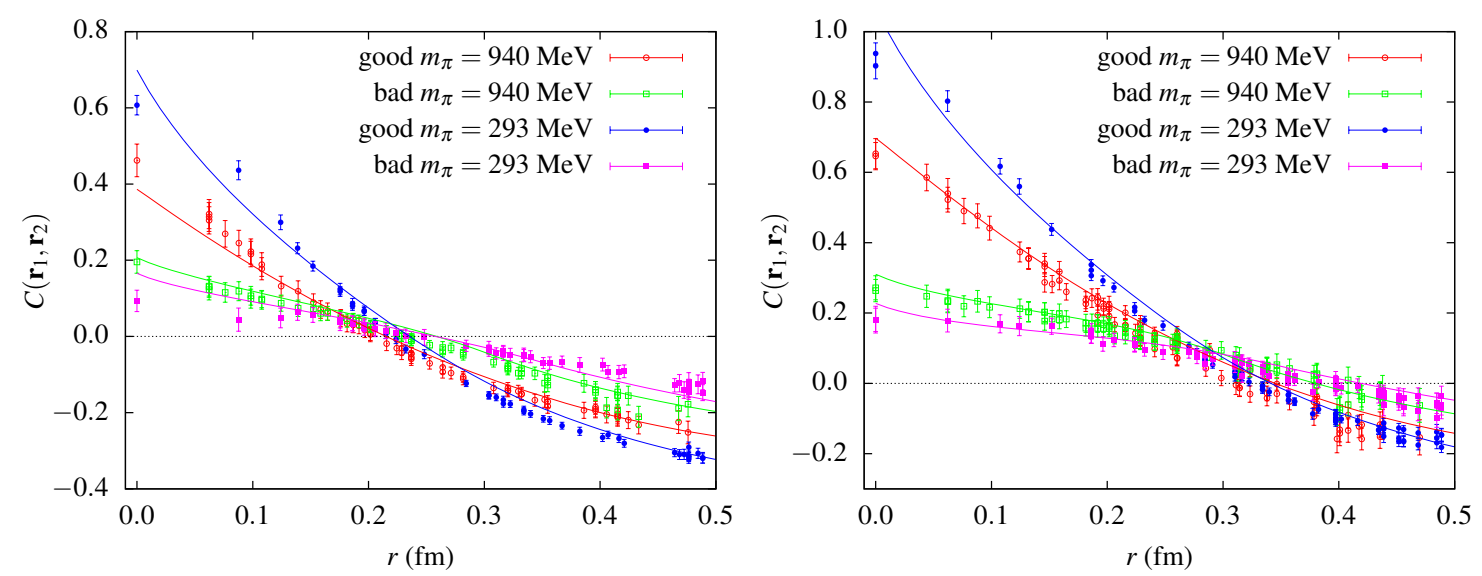

Figure 4: $C\left(\mathbf{r}_{1}, \mathbf{r}_{2}\right)$, as a function of $r$ (in fm) with $R=0.2 \mathrm{fm}$ (left) and $R=0.4 \mathrm{fm}$ (right), with $\mathbf{r} \perp \mathbf{R}$ for the good and bad diquarks and the two pion masses.

static quark, we compared different directions of $\mathbf{r}$. Even at $R=0.2 \mathrm{fm}, C$ was independent of the direction of $\mathbf{r}$, indicating that this correlation function works quite well.

Finally, we can compare the systems. Fig. 4 shows the profile of the correlation function $C$ at two fixed distances $R$ from the static quark to the center of the diquark, and Fig. 5 shows the full dependence of $C$ on $\mathbf{r}$, at fixed $R=0.4 \mathrm{fm}$. The good diquark has a large positive correlation at small $r$ that becomes negative at large $r$. The bad diquark has similar behavior with smaller magnitude. The difference between the good and bad diquarks is larger for the lighter pion mass, as expected from the quark mass dependence of the spin coupling that splits good and bad diquarks. As $R$ increases, both the correlation and the size of the positive region grow, although it is possible that some of this growth of $C$ as $R$ increases may arise from the normalization of the correlation function.

Our main conclusions are seen clearly in Fig. 5. The diquark correlations are highly independent of $\theta$, indicating negligible polarization by the heavy quark, are much stronger in the good rather than the bad channel, and increase strongly with decreasing quark mass. Finally, it is important to note that the diquark radius is approximately $0.3 \mathrm{fm}$ and the hadron half-density radius is also roughly $0.3 \mathrm{fm}$, so the diquark size is comparable to the hadron size. This is reminiscent of the size of Cooper pairs in nuclei, and argues against hadron models requiring point-like diquarks.

\section{Acknowledgments}

This work was supported in part by funds provided by the U.S. Department of Energy under Grants No. DE-FG02-94ER40818 and DE-FG02-96ER40965. P.V. acknowledges support by the MIT Undergraduate Research Opportunities Program (UROP). Additional domain wall propagators were computed using the Chroma software suite [17].

\section{References}

[1] M. Anselmino, E. Predazzi, S. Ekelin, S. Fredriksson and D. B. Lichtenberg, Rev. Mod. Phys. 65 (1993) 1199-1234. 

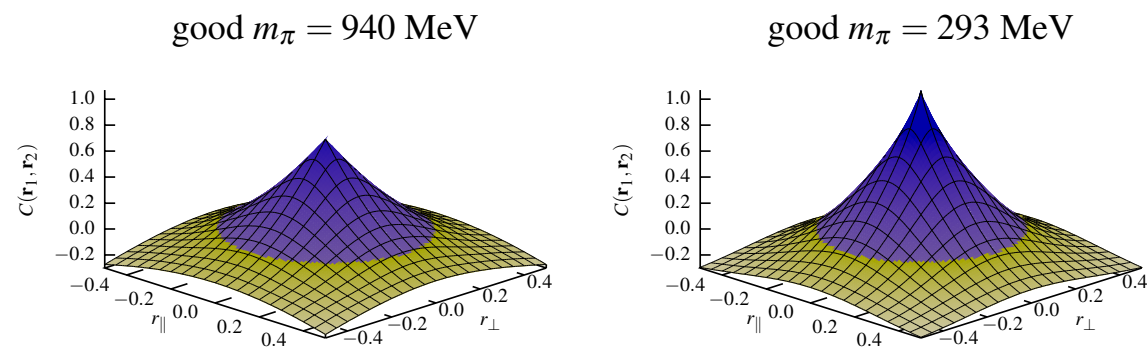

$\operatorname{bad} m_{\pi}=940 \mathrm{MeV}$

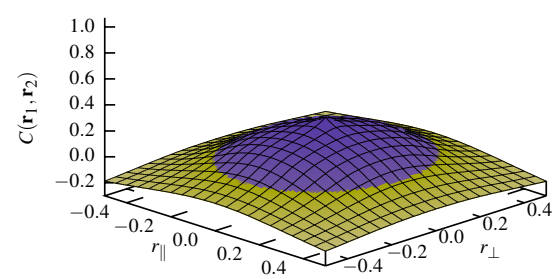

$\operatorname{bad} m_{\pi}=293 \mathrm{MeV}$

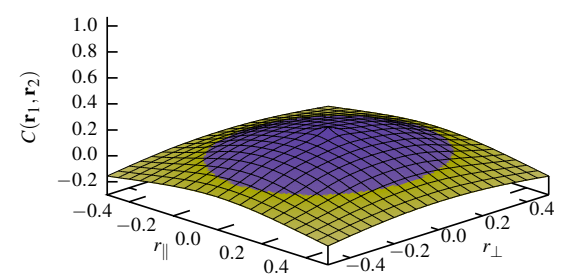

Figure 5: Continuous $C\left(\mathbf{r}_{1}, \mathbf{r}_{2}\right)$ derived from the fit, as a function of $\mathbf{r}$ (in fm) with $R=0.4 \mathrm{fm}$. The two axes $r_{\|}$and $r_{\perp}$ indicate directions of $\mathbf{r}$ parallel to and orthogonal to $\mathbf{R}$, respectively. The color of the surface is discontinuous at $C=0$.

[2] M. Neubert and B. Stech, Phys. Rev. D44 (1991) 775-793.

[3] A. Selem and F. Wilczek, in New trends in HERA physics. Proceedings, Ringberg Workshop, Tegernsee, Germany, October 2-7, 2005, pp. 337-356, 2006. hep-ph / 0602128.

[4] R. L. Jaffe, Phys. Rept. 409 (2005) 1-45.

[5] A. De Rujula, H. Georgi and S. L. Glashow, Phys. Rev. D12 (1975) 147-162.

[6] T. A. DeGrand, R. L. Jaffe, K. Johnson and J. E. Kiskis, Phys. Rev. D12 (1975) 2060.

[7] T. Schäfer and E. V. Shuryak, Rev. Mod. Phys. 70 (1998) 323-426.

[8] R. Babich et. al., Phys. Rev. D76 (2007) 074021.

[9] C. Alexandrou, P. de Forcrand and B. Lucini, Phys. Rev. Lett. 97 (2006) 222002.

[10] M. Burkardt, J. M. Grandy and J. W. Negele, Ann. Phys. 238 (1995) 441-472.

[11] C. Alexandrou, P. de Forcrand and O. Jahn, Nucl. Phys. Proc. Suppl. 119 (2003) 667-669.

[12] T. T. Takahashi, H. Suganuma, Y. Nemoto and H. Matsufuru, Phys. Rev. D65 (2002) 114509.

[13] LHPC Collaboration, J. D. Bratt et. al., Phys. Rev. D (to be published), 1001.3620.

[14] C. W. Bernard et. al., Phys. Rev. D64 (2001) 054506.

[15] G. Kilcup, D. Pekurovsky and L. Venkataraman, Nucl. Phys. Proc. Suppl. 53 (1997) 345-348.

[16] S. Necco and R. Sommer, Nucl. Phys. B622 (2002) 328-346.

[17] SciDAC Collaboration, R. G. Edwards and B. Joó, Nucl. Phys. Proc. Suppl. 140 (2005) 832. 